\title{
Estudio sistemático de las propiedades mecánicas de las micropartículas de magnetita embebidas en una matriz de concreto polimérico
}

\section{Systematic study about the mechanic properties of the magnetite microparticles embedded in a polymeric concrete matrix}

VILLANUEVA-RAYA， Dulce Magdalena ${ }^{1} \uparrow ，$ BÉJAR-BARRIENTOS， Gerardo Enrique ${ }^{1}$, GUERRERO-SERRANO, Azdrubal Lobo ${ }^{2}$ y CABAL-VELARDE, Javier Gustavo ${ }^{1 *}$

${ }^{1}$ Instituto Tecnológico Superior de Irapuato, Carretera Irapuato-Silao km 12.5 C.P. 36821, Irapuato, Gto., México.

${ }^{2}$ Área Académica de Ciencias de la Tierra y Materiales, Universidad Autónoma del Estado de Hidalgo. Carr. PachucaTulancingo Km. 4.5, Col. Campo de Tiro, 42039 Pachuca de Soto, México.

\author{
ID $1^{\text {st }}$ Author: Dulce Magdalena, Villanueva-Raya / ORC ID: 0000-0002-7613-4078, CVU CONACYT ID: 1016131 \\ ID $1^{\text {st }}$ Coauthor: Gerardo Enrique, Béjar-Barrientos / ORC ID: 0000-0001-7986-2532, CVU CONACYT ID: 1016123 \\ ID $2^{\text {do }}$ Coautor: Azdrubal Lobo Guerrero Serrano / ORCID: 0000-0001-5816-847X, CVU CONACYT ID: 171617 \\ ID $3^{\text {er }}$ Coautor: Javier Gustavo, Cabal-Velarde / ORC ID: 0000-0002-5516-3849, CVU CONACYT ID: 99159
}

DOI: $10.35429 / J C E .2019 .9 .3 .12 .16$

Recibido 03 de Junio, 2019; Aceptado 30 Septiembre, 2019

\section{Resumen}

En este trabajo reportamos la síntesis y caracterización de concreto polimérico magnético a base de micropartículas de magnetita embebidas en una matriz de cemento polimérico, así como el estudio sistemático de sus propiedades mecánicas a diferentes concentraciones de micropartículas de cemento y magnetita como material de refuerzo de la matriz polimérica. Este muestreo se efectúo variando la concentración de los reactivos durante el proceso de síntesis para poder analizar de una forma más detallada el cambio de propiedades físicas. La caracterización se llevó a cabo mediante ensayos de tensión y compresión, así como microscopía óptica y electrónica de barrido y XRD. Los resultados obtenidos contribuyen al control sistemático de sus propiedades físicas del concreto polimérico magnético sintetizado con micropartículas de magnetita $\left(\mathrm{Fe}_{3} \mathrm{O}_{4}\right)$ y micropartículas de cemento. Conforme a la concentración mínima de sustancias oleofílicas, el comportamiento mecánico de las muestras es más rígido (plástico). En cambio, cuando se tiene una concentración elevada de sustancias oleofílicas el comportamiento es más elástico (elastómero), ya que presenta mayor desplazamiento. El comportamiento plástico-elástico se presenta a una concentración media de sustancia oleofílica.

Micropartículas, Magnetita, Concreto polimérico

\begin{abstract}
In this paper, we present the synthesis and characterization of magnetic polymeric concrete with microparticles of magnetite embedded in a polymeric concrete matrix. Including the systematic study of the mechanic properties varying the concentration of concrete and magnetite microparticles like the reinforcement of the polymeric matrix. This sampling has been made varying the concentration of the reactive in the process of synthesis to analyze by a detailed way the change of physic properties. This characterization has been made by tension and compression tests, such as the optic microscopic, scanning electronics and XRD. The obtained results contribute to the systematic control of the physics properties about the polymeric magnetic sintered with microparticles of magnetite (Fe3O4) and microparticles of concrete. According to the minimum concentration of oleophilic substances, the mechanic the behavior of the probes is stiffer (plastic). Instead when there has a high concentration of oleophilic substance the behavior is more elastic (elastomer) because the probe presents more displacement. The behavior plastic-elastic is presented in a medium concentration of oleophilic substance.
\end{abstract}

Microparticles, Magnetite, Polymeric concrete

Citación: VILLANUEVA-RAYA, Dulce Magdalena, BÉJAR-BARRIENTOS, Gerardo Enrique1, GUERREROSERRANO, Azdrubal Lobo y CABAL-VELARDE, Javier Gustavo. Estudio sistemático de las propiedades mecánicas de las micropartículas de magnetita embebidas en una matriz de concreto polimérico. Revista de Ingeniería Civil. 2019. 3-9: 12-16

\footnotetext{
$\dagger$ Investigador contribuyendo como primer autor.
} 


\section{Introducción}

Día con día el ser humano busca satisfacer sus necesidades, para ello necesita emplear diversos materiales, cada material tiene características y propiedades distintas. Conforme a la necesidad a satisfacer se emplea el material con las propiedades correctas. Hoy en día, por medio de estudios e investigaciones se han desarrollado compositos con una mayor durabilidad y resistencia, donde su eficiencia es mayor en comparación a otros materiales. Por otra parte, la micro y nanotecnología ha sido un área de investigación muy interesante por la parte de los materiales.

Un composito es un material compuesto por una matriz, que puede ser de naturaleza orgánica o inorgánica a la cual se le adiciona un material que puede ser de cohesión o de refuerzo (comúnmente llamado "material de relleno") y que se encuentra en mucha menor proporción. Usualmente ambos materiales, la matriz y el refuerzo-relleno, son inmiscibles entre sí y lo que ocurre es la dispersión del refuerzo en la matriz, con lo cual al relleno se lo denomina también como fase dispersa. Un mismo composito puede estar compuesto con dos o más fases dispersas, que se clasifican en función de la forma, tamaño distribución y orientación del material de relleno. Al combinar el material de la matriz con el material de relleno, se obtiene un material con nuevas propiedades físicas y químicas. [1]

Las nanopartículas magnéticas han atraído un interés considerable, principalmente magnetita y maghemita, debido, no solo a sus propiedades magnéticas, sino también su estabilidad química y sus propiedades magnetoeléctricas.[2, 3] Otra de las cualidades que poseen las nanopartículas magnéticas de pequeño tamaño es el superparamagnétismo. Esto ocurre debido a que las nanopartículas presentan un dominio magnético único y un ciclo de histéresis coercitivo igual o muy próximo a cero. En este estado las nanopartículas magnéticas son capaces de seguir a un imán y a su vez perder la magnetización al eliminar el campo magnético.[4] La fuerza magnética generada por una sola nanopartícula e débil e impide la respuesta inmediata al campo magnético. Esto debido a que cuando la nanopartícula se mueve a lo largo de una solución se genera una resistencia hidrodinámica.
Ahora bien, si la nanopartícula es mayor, o mejor aún, se dispone de un "clúster" de nanopartículas en una matriz polimérica lo que se conoce como micropartículas magnéticas entonces la resistencia hidrodinámica se reduce y la respuesta al campo magnético se ve aumentada. [5, 6]

Las micropartículas magnéticas siguen teniendo el mismo comportamiento superparamagnético que las nanopartículas, debido a que no ocurren interacciones magnéticas entre las distintas nanopartículas magnéticas en su interior. Por otro lado, el componente polimérico estabiliza las nanopartículas magnéticas en su interior, además de dotarlas de grupos funcionales necesarios para diversas aplicaciones. [7]

En este caso se realizó un estudio sistemático de un tipo de concreto polimérico compuesto por silicón, cemento y sustancias oleofílicas, a su vez es reforzado con micropartículas de magnetita. Se pretende encontrar algún efecto en el concreto polimérico haciendo variaciones en la concentración de los reactivos que componen el material para observar si hay una mejora en cuanto a propiedades físicas. La importancia de dicho estudio es analizar las propiedades que el material presenta para determinar su eficiencia.

\section{Materiales}

$\begin{array}{ll}- & \text { Silicón (EPSA) } \\ - & \text { Aceite (EPSA) } \\ - & \text { Cemento (Cemex) } \\ - & \text { Catalizador } \\ - & \text { Micropartículas de magnetita }\end{array}$

\section{Metodología}

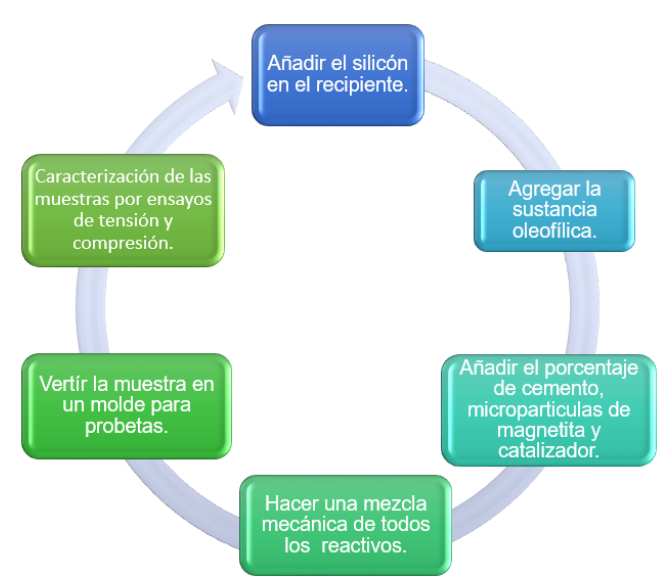

Figura 1 Diagrama de flujo que muestra la metodología de las muestras 


\section{Resultados}

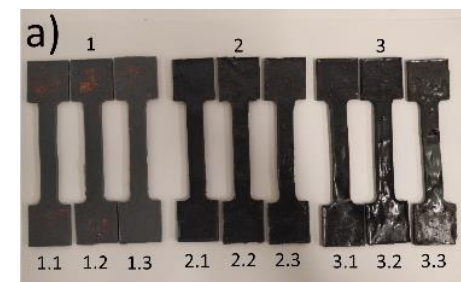

b)

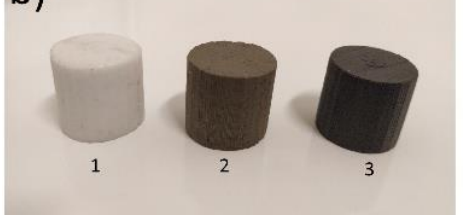

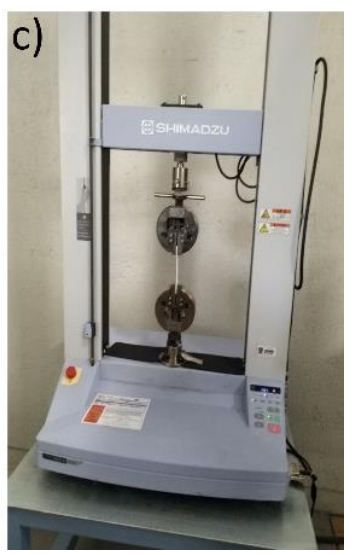

Figura 2 a) Muestras a diferentes concentraciones de reactivos para ensayo de tensión. b) Muestras de silicón, silicón-cemento, silicón-cemento-micropartículas para ensayos de compresión. c) Maquina universal

La caracterización mecánica de las muestras a distintas concentraciones de los reactivos que conforman el concreto polimérico magnético se realizaron por medio de la maquina universal que se puede apreciar en la figura 2 (c).

Como se muestra en la figura 2 (a) se observan las probetas realizadas donde las tres muestras correspondientes al grupo 1 contienen un $20 \%$ de sustancia oleofílica respecto al silicón, el grupo dos un 50\% y por consiguiente el grupo tres con $100 \%$ de esta misma sustancia. Para cada grupo de estas probetas también existe una variación del $20 \%, 50 \%$ y $100 \%$ con respecto al cemento y a las micropartículas de magnetita. Por otro lado, en la figura 2 (b) se muestra los cilindros de silicón, silicón-cemento y silicón-cemento-micropartículas, todas con un $100 \%$ de cada reactivo, cada muestra fue sometida a un ensayo de compresión.

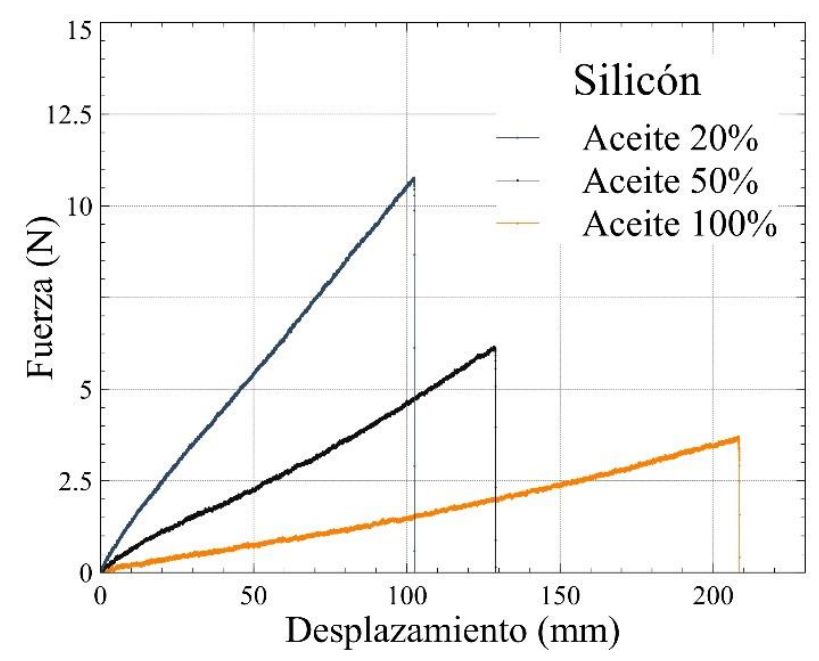

Figura 3 Gráfica de ensayo por tensión de las muestras de silicón
En la figura 3 perteneciente a la gráfica de fuerza contra desplazamiento se muestra el ensayo realizado a una probeta de silicón y sustancia oleofílica donde se pueden observar distintos comportamientos mecánicos dependiendo de la concentración de sustancia oleofílica, cuando mayor sea la cantidad de está, la muestra tenderá a comportarse como un elastómero ya que su desplazamiento es mayor, por otro lado cuando menor sea la cantidad de sustancia oleofílica el material tendrá un comportamiento de un plástico rígido $\mathrm{y}$ soportara más la fuerza aplicada al material.

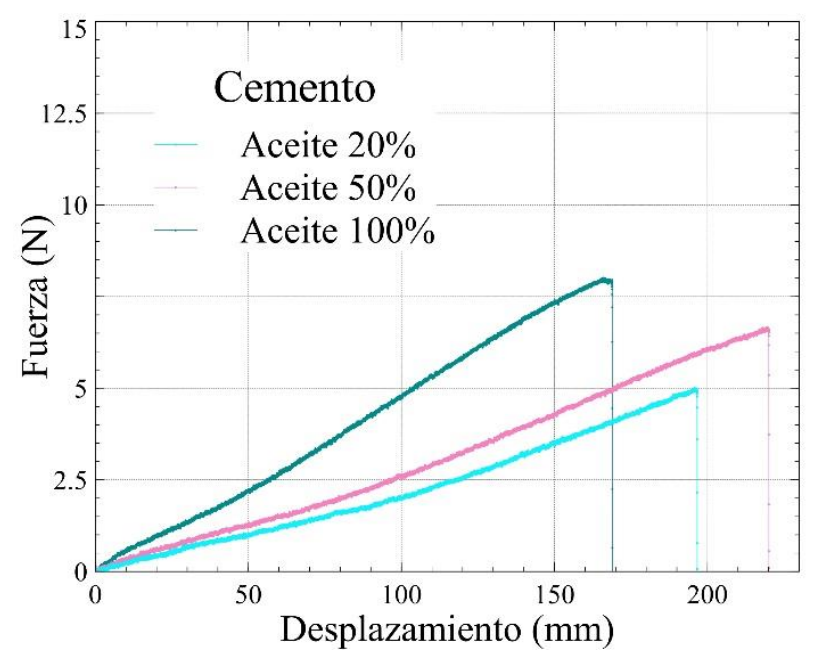

Figura 4 Gráfica de ensayo por tensión de las muestras de silicón y cemento a distintas concentraciones de sustancias oleofílicas

Se puede apreciar en la figura 4, la gráfica donde se muestran las curvas de las muestras de silicón con cemento cabe recalcar que tienen un comportamiento muy distinto ya que las muestras presentan un mayor desplazamiento en comparación con las muestras que solamente contienen silicón. En el caso de la muestra que contiene el mayor porcentaje de sustancias oleofílicas la muestra presenta una mayor resistencia y desplazamiento con el refuerzo de cemento en comparación con la curva de mayor porcentaje de sustancia. 


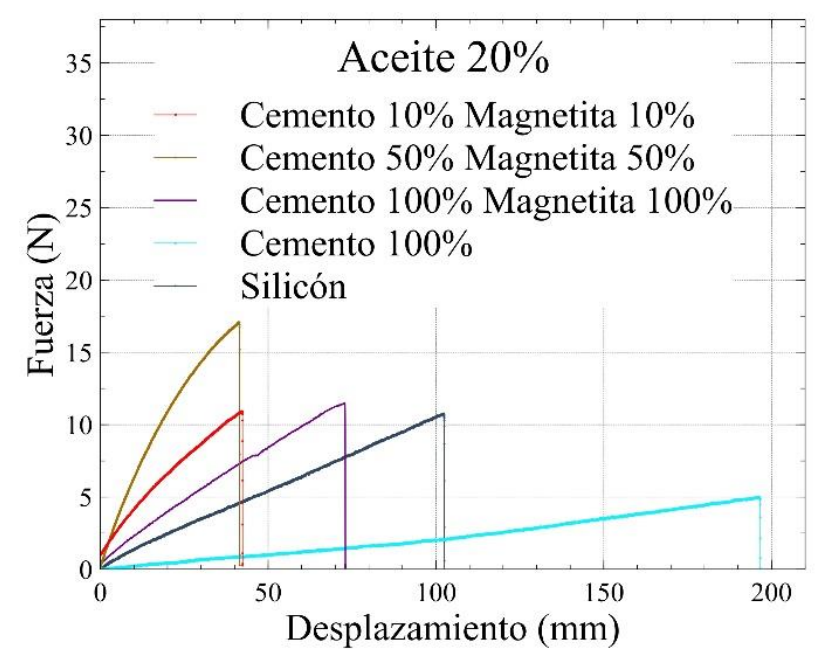

Figura 5 Gráfica de ensayo por tensión de las muestras que contienen cemento, micropartículas de magnetita, silicón, adicionando las curvas de la muestra de cemento con silicón y la muestra de silicón, todas a una concentración del $20 \%$ de sustancia oleofílica

En cuanto a la figura 5, se puede apreciar un gran cambio en las curvas de las muestras que tienen como refuerzo el cemento y las micropartículas de magnetita, ya que presentan un comportamiento más rígido por lo que las hace más resistentes, sin embargo, la curva que menos resiste es la que contiene solamente cemento como refuerzo, es la curva que presenta un comportamiento de un elastómero. Cabe recalcar que la muestra que no contiene algún refuerzo presenta desplazamiento y resistencia a la fuerza aplicada. Esta gráfica pertenece al muestreo de baja concentración de sustancias oleofílicas

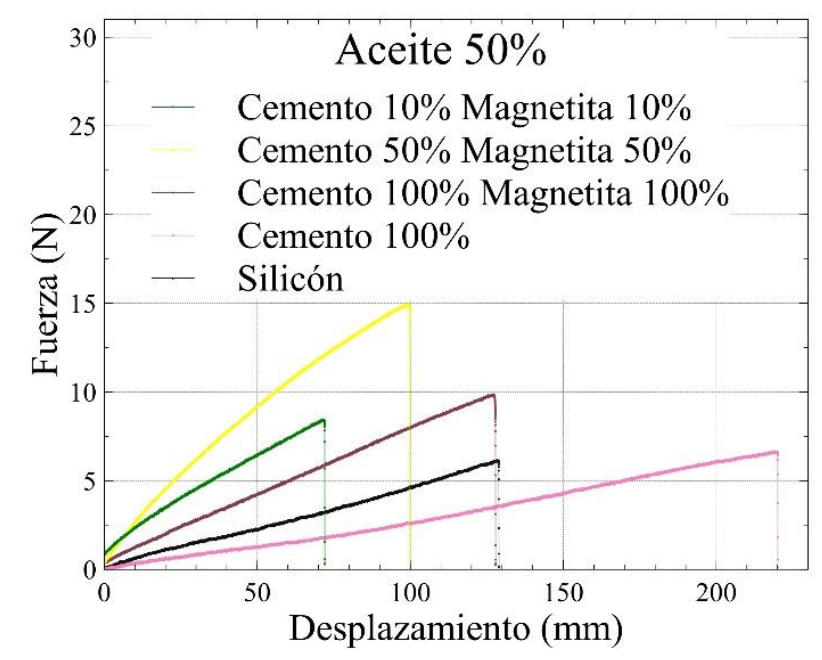

Figura 6 Gráfica de ensayo por tensión de las muestras que contienen cemento, micropartículas de magnetita, silicón, adicionando las curvas de la muestra de cemento con silicón y la muestra de silicón, todas a una concentración del $50 \%$ de sustancia oleofílica
Se aprecia que en la gráfica de fuerza contra desplazamiento de la figura 6, las muestras presentan mayor desplazamiento respecto a las muestras de la figura 5 , esto era esperable ya que se aumentó la concentración de porcentaje de la sustancia oleofílica, se observó que la muestra de solo silicón y cemento es la que sigue manteniendo con mayor desplazamiento respecto a las demás muestras.

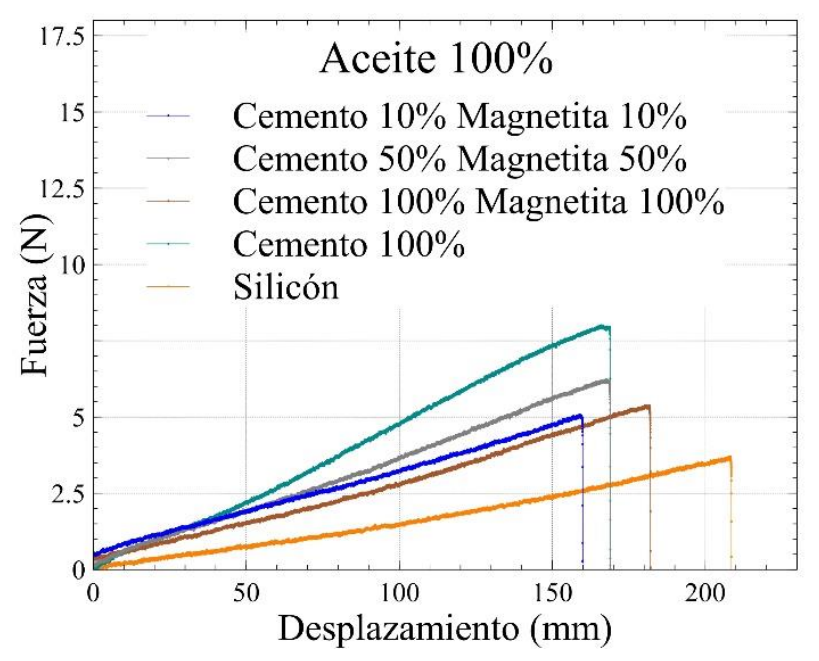

Figura 7 Gráfica de ensayo por tensión de las muestras que contienen cemento, micropartículas de magnetita, silicón, adicionando las curvas de la muestra de cemento con silicón y la muestra de silicón, todas a una concentración del $100 \%$ de sustancia oleofílica

En el caso de la figura 7 la gráfica de fuerza contra deformación muestra un gran cambio respecto a las otras gráficas de las figuras 5 y 6 , ya que la muestra que contiene silicón y cemento al $100 \%$ al igual que la concentración de sustancia oleofílica, es la muestra que presenta una mayor resistencia a la fuerza aplicada. Se realizó una comparación de las gráficas de la figura 5, 6 y 7 observando las muestras que contienen micropartículas de magnetita a distintas concentraciones, dando como resultado de ello que la resistencia de las muestras es proporcionada por las micropartículas de magnetita. 


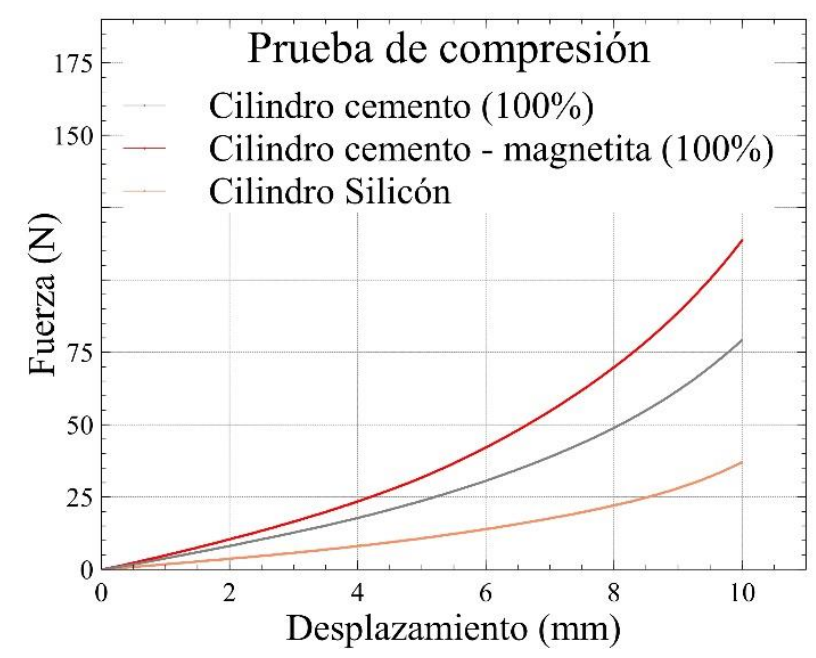

Figura 8 Curvas de ensayo por compresión de las muestras de silicón, cemento-silicón, cemento-silicónmicropartículas de magnetita

La figura 8 presenta las gráficas de fuerza contra desplazamiento de las muestras de silicón, silicón-cemento y silicón-cemento y micropartículas, donde se recalca lo mencionado anteriormente; la muestra que contiene solo silicón es la que presenta un mayor desplazamiento mostrando ser más deformables por la fuerza aplicada. La muestra de silicóncemento presentan ser menos deformables lo que indica que el cemento le da rigidez al material. La mayor rigidez se presenta en las muestras que contienen silicón-cemento y micropartículas de magnetita, por lo que el material de refuerzo en nuestro material son las micropartículas.

\section{Conclusiones}

De acuerdo los resultados preliminares se obtuvieron los estudios sistemáticos del análisis de las propiedades mecánicas del comportamiento a diferentes concentraciones de los reactivos, obteniendo las curvas de esfuerzo contra deformación tanto de ensayos de tensión como por ensayos de compresión. Gracias a estas curvas se puede observar un comportamiento distinto en sus propiedades mecánicas en cada una de las muestras de acuerdo con la variación de concentración con respecto a los reactivos que conforman la matriz del concreto polimérico.

\section{Referencias}

[1] Ruíz, M.M. Estudio de compositos magnetoelásticos estructurados. Síntesis y caracterización de partículas magnéticas y conductoras para obtener propiedades de Piezo y Magneto resistencia. 2016.
[2] Li, Q., et al., Correlation between particle sizeldomain structure and magnetic properties of highly crystalline Fe3O4 nanoparticles. Scientific Reports, 2017. 7(1): p. 9894.

[3] Yoo, K., et al., Quantitative Measurements of Size-Dependent Magnetoelectric Coupling in Fe3O4 Nanoparticles. Nano Letters, 2016. 16(12): p. 7408-7413.

[4] Gutiérrez, L., et al., Aggregation effects on the magnetic properties of iron oxide colloids. Nanotechnology, 2019. 30(11): p. 112001.

[5] Lu, Y., et al., Biogenic and biomimetic magnetic nanosized assemblies. Nano Today, 2012. 7(4): p. 297-315.

[6] Llamedo González, A., Síntesis y caracterización de micropartículas magnéticas con aplicaciones en nanomedicina. 2019.

[7] Tudorache, M., A. Tencaliec, and C. Bala, Magnetic beads-based immunoassay as asensitive alternative for atrazine analysis. Talanta, 2008. 77(2): p. 839-843. 